Surface-state-induced second-harmonic generation

This article has been downloaded from IOPscience. Please scroll down to see the full text article.

1996 J. Phys.: Condens. Matter 86629

(http://iopscience.iop.org/0953-8984/8/36/015)

The Table of Contents and more related content is available

Download details:

IP Address: 140.112.113.225

The article was downloaded on 17/07/2009 at 07:34

Please note that terms and conditions apply. 


\title{
Surface-state-induced second-harmonic generation
}

\author{
C D Hu \\ Department of Physics, National Taiwan University, Taipei, Taiwan, Republic of China
}

Received 17 April 1996

\begin{abstract}
We propose a simple and intuitively clear method for analysing the second-harmonic generation induced by surface states. The two-band model is used to calculate the wave functions and Green's function so that the second-harmonic current density and polarization can be evaluated. Assuming that the polarization is concentrated on the surface, we calculated the reflection intensity of the second-harmonic light. We apply our formalism to the L-gap surface states on the silver (110) surface and obtain results that agree reasonably well with experiments.
\end{abstract}

\section{Introduction}

Second-harmonic generation is not only a very useful tool for surface diagnostics, but is itself a physically interesting phenomenon [1]. In the past few years, there have been several experimental works on the surface-state-related second-harmonic generation [2-4] on noblemetal surfaces. By analysing the relationship between the second-harmonic intensity and frequency, incident angle and polarization, one is able to calculate accurately the energy, and dispersion, and find the symmetry of the surface states involved. Other information such as the energy gap where the surface states are located, surface characterization and dielectric function may also be obtained. Therefore, it is desirable to have a physical understanding and quantitative analysis of this topic. The second-harmonic generation on jellium surfaces had been studied thoroughly [5-8]. But, for a system with energy-gap surface states, there is no comparable work. This article is an attempt to fill this void.

One of the difficulties of analysing the problem of the second-harmonic generation is that there are infinitely many intermediate states to account for. Usually one can choose a set of states which provide the main contribution-for an example, resonant states. For a system with an energy gap this is not trivial because sometimes the energy range of resonance falls into the gap. It is not clear that the states near the band edge (and hence those closest to resonance) give the dominant contribution. The states further away, though they contribute less individually, are greater in number. The entire band has to be taken into account; at least convergence has to be reached. As we shall see, the Green's function method can overcome this difficulty. However, we do not intend to consider the manyelectron effect which was treated in references [5-8]. The future challenge is to incorporate both this and the band-gap effect into one formalism. We are working on this subject.

In section 2, we derive the form of the second-harmonic current density in terms of the Green's function. In section 3, we use the two-band model to compute the wave functions of the surface states and the Green's function. The latter is a modified version of that in Mahan's photoemission theory [9]. Since the energy gap is the main feature in our system, it has to be incorporated in our calculation of the Green's function. We did not attempt to use 
a more sophisticated band-structure method. The two-band model [10] gives a reasonably good description of the energy gap and surface states and it is intuitively simple. Thus it is easier to introduce the many-body effect, which is the subject of our future work. Also, the present approach can be readily extended to more sophisticated methods such as the KKR method. In section 4, we compute the second-harmonic reflectance using the model of Sipe and co-workers [11] and a simple dielectric function which fits the experimental data. The silver L gap on the (110) surface is used as an example. We give conclusions in section 5.

\section{Formalism}

We derive the form of the second-harmonic current density in this section. It is similar to that in Bower's approach [12] and had been derived before [13, 14]. The Hamiltonian of the electron-EM-wave interaction is

$$
H_{\text {int }}=\int \mathrm{d}^{3} \boldsymbol{r}\left(h_{1}+h_{2}\right)
$$

where

$$
h_{1}=\frac{-e}{2 m c} \psi^{\dagger}(\boldsymbol{r})[\boldsymbol{p} \cdot \boldsymbol{A}(\boldsymbol{r}, t)+\boldsymbol{A}(\boldsymbol{r}, t) \cdot \boldsymbol{p}] \psi(\boldsymbol{r})
$$

and

$$
h_{2}=\frac{e^{2}}{2 m c^{2}} \hat{\Psi}^{\dagger}(\boldsymbol{r}) \boldsymbol{A}^{2}(\boldsymbol{r}, t) \hat{\psi}(\boldsymbol{r})
$$

Here $\hat{\psi}(\boldsymbol{r})$ is the electron field operator, the light inside the crystal is represented by the vector potential $\boldsymbol{A}(\boldsymbol{r}, t)$ and

$$
\boldsymbol{A}(\boldsymbol{r}, t)=\boldsymbol{A}_{0} \mathrm{e}^{\mathrm{i}(\boldsymbol{u} \cdot \boldsymbol{r}-\omega t)}
$$

where $\boldsymbol{u}$ is the wave vector. We have used gauge where

$$
\boldsymbol{E}=-\frac{1}{c} \frac{\partial \boldsymbol{A}}{\partial t}
$$

Using the time-dependent perturbation theory we found that the current density to the second order in $\boldsymbol{A}_{0}$ is

$$
\boldsymbol{J}(\boldsymbol{r}, t)=\boldsymbol{J}_{1}(\boldsymbol{r}, t)+\boldsymbol{J}_{2}(\boldsymbol{r}, t)+\boldsymbol{J}_{3}(\boldsymbol{r}, t)
$$

with

$$
\begin{aligned}
& \boldsymbol{J}_{1}(\boldsymbol{r}, t)=-\sum_{n} \int \mathrm{d}^{3} \boldsymbol{r}^{\prime}\left\{\frac{\left[h_{1}\left(\boldsymbol{r}^{\prime}, t\right)\right]_{0 n}\left[\boldsymbol{J}_{A}(\boldsymbol{r})\right]_{n 0}}{\hbar \omega+E_{n 0}+\mathrm{i} \eta}-\frac{\left[\boldsymbol{J}_{A}(\boldsymbol{r})\right]_{0 n}\left[h_{1}\left(\boldsymbol{r}^{\prime}, t\right)\right]_{n 0}}{\hbar \omega-E_{n 0}+\mathrm{i} \eta}\right\} \\
& \boldsymbol{J}_{2}(\boldsymbol{r}, t)=-\sum_{n} \int \mathrm{d}^{3} \boldsymbol{r}^{\prime}\left\{\frac{\left[h_{2}\left(\boldsymbol{r}^{\prime}, t\right)\right]_{0 n}\left[\boldsymbol{J}_{p}(\boldsymbol{r})\right]_{n 0}}{2 \hbar \omega+E_{n 0}+\mathrm{i} \eta}-\frac{\left[\boldsymbol{J}_{p}(\boldsymbol{r})\right]_{0 n}\left[h_{2}\left(\boldsymbol{r}^{\prime}, t\right)\right]_{n 0}}{2 \hbar \omega-E_{n 0}+\mathrm{i} \eta}\right\}
\end{aligned}
$$

and

$$
\begin{aligned}
\boldsymbol{J}_{3}(\boldsymbol{r}, t)=- & \sum_{m, n} \int \mathrm{d}^{3} \boldsymbol{r}^{\prime} \int \mathrm{d}^{3} \boldsymbol{r}^{\prime \prime}\left\{\frac{\left[\boldsymbol{J}_{p}(\boldsymbol{r})\right]_{0 n}\left[h_{1}\left(\boldsymbol{r}^{\prime}, t\right)\right]_{n m}\left[h_{1}\left(\boldsymbol{r}^{\prime \prime}, t\right)\right]_{m 0}}{\left(2 \hbar \omega-E_{n 0}+\mathrm{i} \eta\right)\left(\hbar \omega-E_{m 0}+\mathrm{i} \eta\right)}\right. \\
& -\frac{\left[h_{1}\left(\boldsymbol{r}^{\prime}, t\right)\right]_{0 n}\left[\boldsymbol{J}_{p}(\boldsymbol{r})\right]_{n m}\left[h_{1}\left(\boldsymbol{r}^{\prime \prime}, t\right)\right]_{m 0}}{\left(\hbar \omega+E_{n 0}+\mathrm{i} \eta\right)\left(\hbar \omega-E_{m 0}+\mathrm{i} \eta\right)} \\
& \left.+\frac{\left[h_{1}\left(\boldsymbol{r}^{\prime \prime}, t\right)\right]_{0 n}\left[h_{1}\left(\boldsymbol{r}^{\prime}, t\right)\right]_{n m}\left[\boldsymbol{J}_{p}(\boldsymbol{r})\right]_{m 0}}{\left(2 \hbar \omega+E_{n 0}+\mathrm{i} \eta\right)\left(\hbar \omega+E_{m 0}+\mathrm{i} \eta\right)}\right\}
\end{aligned}
$$


where $\eta$ is the line width. We have defined

$$
\boldsymbol{J}_{p}(\boldsymbol{r}) \equiv \frac{e}{2 m}\left\{\hat{\psi}^{\dagger}(\boldsymbol{r}) \boldsymbol{p} \hat{\psi}(\boldsymbol{r})-\left[\boldsymbol{p} \hat{\psi}^{\dagger}(\boldsymbol{r})\right] \hat{\psi}(\boldsymbol{r})\right\}
$$

and

$$
\boldsymbol{J}_{A}(\boldsymbol{r}) \equiv-\frac{e^{2}}{m^{2} c} \hat{\psi}^{\dagger}(\boldsymbol{r}) \boldsymbol{A}(\boldsymbol{r}, t) \hat{\psi}(\boldsymbol{r})
$$

The above expressions can be simplified if we consider only the vertical transition-i.e., neglect the momentum of light because it is much smaller than that of electrons. As a result,

$$
\begin{aligned}
\boldsymbol{J}_{1}(\boldsymbol{r}, t)=\frac{e^{3}}{m^{3} c^{2}} \sum_{n} \int \mathrm{d}^{3} \boldsymbol{r}^{\prime}\left\{\frac{\left[\hat{\psi}^{\dagger}\left(\boldsymbol{r}^{\prime}\right) \boldsymbol{A}\left(\boldsymbol{r}^{\prime}, t\right) \cdot \boldsymbol{p}^{\prime} \hat{\psi}\left(\boldsymbol{r}^{\prime}\right)\right]_{0 n}\left[\hat{\psi}^{\dagger}(\boldsymbol{r}) \boldsymbol{A}(\boldsymbol{r}, t) \hat{\psi}(\boldsymbol{r})\right]_{n 0}}{\hbar \omega+E_{n 0}+\mathrm{i} \eta}\right. \\
\left.-\frac{\left[\hat{\psi}^{\dagger}(\boldsymbol{r}) \boldsymbol{A}(\boldsymbol{r}, t) \hat{\psi}(\boldsymbol{r})\right]_{0 n}\left[\hat{\psi}^{\dagger}\left(\boldsymbol{r}^{\prime}\right) \boldsymbol{A}\left(\boldsymbol{r}^{\prime}, t\right) \cdot \boldsymbol{p}^{\prime} \hat{\psi}\left(\boldsymbol{r}^{\prime}\right)\right]_{n 0}}{\hbar \omega-E_{n 0}+\mathrm{i} \eta}\right\} \\
\boldsymbol{J}_{2}(\boldsymbol{r}, t)=-\frac{e^{3}}{2 m^{3} c^{2}} \sum_{n} \int \mathrm{d}^{3} \boldsymbol{r}^{\prime} \boldsymbol{A}^{2}\left(\boldsymbol{r}^{\prime}, t\right)\left\{\frac{\left[\hat{\psi}^{\dagger}\left(\boldsymbol{r}^{\prime}\right) \hat{\psi}\left(\boldsymbol{r}^{\prime}\right)\right]_{0 n}\left[\hat{\psi}^{\dagger}(\boldsymbol{r}) \boldsymbol{p} \hat{\psi}(\boldsymbol{r})\right]_{n 0}}{2 \hbar \omega+E_{n 0}+\mathrm{i} \eta}\right. \\
\left.-\frac{\left[\hat{\psi}^{\dagger}(\boldsymbol{r}) \boldsymbol{p} \hat{\psi}(\boldsymbol{r})\right]_{0 n}\left[\hat{\psi}^{\dagger}\left(\boldsymbol{r}^{\prime}\right) \hat{\psi}\left(\boldsymbol{r}^{\prime}\right)\right]_{n 0}}{2 \hbar \omega-E_{n 0}+\mathrm{i} \eta}\right\}
\end{aligned}
$$

and

$$
\begin{aligned}
\boldsymbol{J}_{3}(\boldsymbol{r}, t)=-\frac{e^{3}}{m^{3} c^{2}} \sum_{m, n, i, j} \int \mathrm{d}^{3} \boldsymbol{r}^{\prime} \int \mathrm{d}^{3} \boldsymbol{r}^{\prime \prime} A_{i}\left(\boldsymbol{r}^{\prime}, t\right) A_{j}\left(\boldsymbol{r}^{\prime \prime}, t\right) \\
\quad \times\left\{\frac{\left[\hat{\psi}^{\dagger}(\boldsymbol{r}) \boldsymbol{p} \hat{\psi}(\boldsymbol{r})\right]_{0 n}\left[\hat{\psi}^{\dagger}\left(\boldsymbol{r}^{\prime}\right) p_{i}^{\prime} \hat{\psi}\left(\boldsymbol{r}^{\prime}\right)\right]_{n m}\left[\hat{\psi}^{\dagger}\left(\boldsymbol{r}^{\prime \prime}\right) p_{j}^{\prime \prime} \hat{\psi}\left(\boldsymbol{r}^{\prime \prime}\right)\right]_{m 0}}{\left(2 \hbar \omega-E_{n 0}+\mathrm{i} \eta\right)\left(\hbar \omega-E_{m 0}+\mathrm{i} \eta\right)}\right. \\
-\frac{\left[\hat{\psi}^{\dagger}\left(\boldsymbol{r}^{\prime}\right) p_{i}^{\prime} \hat{\psi}\left(\boldsymbol{r}^{\prime}\right)\right]_{0 n}\left[\hat{\psi}^{\dagger}(\boldsymbol{r}) \boldsymbol{p} \hat{\psi}(\boldsymbol{r})\right]_{n m}\left[\hat{\psi}^{\dagger}\left(\boldsymbol{r}^{\prime \prime}\right) p_{j}^{\prime \prime} \hat{\psi}\left(\boldsymbol{r}^{\prime \prime}\right)\right]_{m 0}}{\left(\hbar \omega+E_{n 0}+\mathrm{i} \eta\right)\left(\hbar \omega-E_{m 0}+\mathrm{i} \eta\right)} \\
\left.+\frac{\left[\hat{\psi}^{\dagger}\left(\boldsymbol{r}^{\prime \prime}\right) p_{j}^{\prime \prime} \hat{\psi}\left(\boldsymbol{r}^{\prime \prime}\right)\right]_{0 n}\left[\hat{\psi}^{\dagger}\left(\boldsymbol{r}^{\prime}\right) p_{i}^{\prime} \hat{\psi}\left(\boldsymbol{r}^{\prime}\right)\right]_{n m}\left[\hat{\psi}^{\dagger}(\boldsymbol{r}) \boldsymbol{p} \hat{\psi}(\boldsymbol{r})\right]_{m 0}}{\left(2 \hbar \omega+E_{n 0}+\mathrm{i} \eta\right)\left(\hbar \omega+E_{m 0}+\mathrm{i} \eta\right)}\right\} .
\end{aligned}
$$

We have to sum over all of the intermediate states for $\boldsymbol{J}_{3}(\boldsymbol{r}, t)$. This can be done by utilizing the Green's function

$$
G\left(\boldsymbol{r}, \boldsymbol{r}^{\prime} ; E\right)=\sum_{n} \frac{\psi_{n}(\boldsymbol{r}) \psi_{n}^{*}\left(\boldsymbol{r}^{\prime}\right)}{E-E_{n}} .
$$

Assuming the ground-state wave function and energy to be $\psi_{0}^{*}(\boldsymbol{r})$ and $E_{0}$, we have

$$
\begin{aligned}
\boldsymbol{J}_{3}(\boldsymbol{r}, t)=\frac{-e^{3}}{m^{3} c^{2}} & \sum_{i, j} \int \mathrm{d}^{3} \boldsymbol{r}^{\prime} \int \mathrm{d}^{3} \boldsymbol{r}^{\prime \prime} A_{i}\left(\boldsymbol{r}^{\prime}, t\right) A_{j}\left(\boldsymbol{r}^{\prime \prime}, t\right) \\
& \times\left[\psi_{0}^{*}(\boldsymbol{r}) \boldsymbol{p} G\left(\boldsymbol{r}, \boldsymbol{r}^{\prime} ; 2 \hbar \omega+E_{0}+\mathrm{i} \eta\right) p_{i}^{\prime} G\left(\boldsymbol{r}^{\prime}, \boldsymbol{r}^{\prime \prime} ; \hbar \omega+E_{0}+\mathrm{i} \eta\right) p_{j}^{\prime \prime} \psi_{0}^{*}\left(\boldsymbol{r}^{\prime \prime}\right)\right. \\
& +\psi_{0}^{*}\left(\boldsymbol{r}^{\prime}\right) p_{i}^{\prime} G\left(\boldsymbol{r}^{\prime}, \boldsymbol{r} ;-\hbar \omega+E_{0}-\mathrm{i} \eta\right) \boldsymbol{p} G\left(\boldsymbol{r}, \boldsymbol{r}^{\prime \prime} ; \hbar \omega+E_{0}+\mathrm{i} \eta\right) p_{j}^{\prime \prime} \psi_{0}^{*}\left(\boldsymbol{r}^{\prime \prime}\right) \\
& \left.+\psi_{0}^{*}\left(\boldsymbol{r}^{\prime \prime}\right) p_{j}^{\prime \prime} G\left(\boldsymbol{r}^{\prime \prime}, \boldsymbol{r}^{\prime} ;-2 \hbar \omega+E_{0}-\mathrm{i} \eta\right) p_{i}^{\prime} G\left(\boldsymbol{r}^{\prime}, \boldsymbol{r} ;-\hbar \omega+E_{0}-\mathrm{i} \eta\right) \boldsymbol{p} \psi_{0}(\boldsymbol{r})\right] .
\end{aligned}
$$

Equations (15) and (16) combined form the basis of our later calculation. 
Table 1. The parameters of the JJJ potential [10]. Only $z_{0}$ is adjusted to make the energies fit with the experimental data.

\begin{tabular}{lllll}
\hline State parity & $U_{0}(\mathrm{Ryd})$ & $\lambda(\mathrm{au})$ & $z_{0}(\mathrm{au})$ & Energy $(\mathrm{eV})$ \\
\hline Odd & 1.03 & 0.96 & 1.78 & -0.1 \\
Even & 1.03 & 0.96 & 2.63 & 1.66 \\
\hline
\end{tabular}

\section{Surface states}

The formalism in the last section will now be applied to the surface-state-induced secondharmonic generation. There are well-characterized surface states on most noble-metal surfaces. So it is convenient to take them as examples and compare the results with experiments. To make calculation simple we use the two-band model. It has been shown that the two-band model is able to provide a good description of surface and image states on noble-metal surfaces $[10,15]$. A more sophisticated model will incorporate more plane waves for the wave function and more Fourier components of the crystal potential. However, the procedure of the calculation remains the same. Furthermore, since we intended to study the high-symmetry plane, the components of the large-crystal reciprocal lattice are less important. The two-band model, though less accurate, retains the main features of this problem. On the silver (110) surface, there are two surface states in the L gap at the $\bar{Y}$ point. The one with lower energy is of odd parity in the $y$-direction and the other is of even parity. The lower state is about $0.1 \mathrm{eV}$ below the Fermi level and therefore is occupied. We set the surface normal to be the $z$-direction and (001) to be the $y$-direction. The reciprocallattice vector $G$ is equal to $(2 \pi / a)(111)$ where $a$ is the lattice constant. The wave functions and their explicit forms inside the crystal are

$\psi_{1}(\boldsymbol{r})=\sqrt{\frac{2}{\mathcal{A}}} \sin \left(G_{y} y / 2\right) Z_{1}(z)=\sqrt{\frac{2}{\mathcal{A}}} \sin \left(G_{y} y / 2\right) c_{z 1} \mathrm{e}^{q_{1} z} \sin \left(G_{z} z / 2+\delta_{1}\right) \quad z<0$

and

$\psi_{2}(\boldsymbol{r})=\sqrt{\frac{2}{\mathcal{A}}} \cos \left(G_{y} y / 2\right) Z_{2}(z)=\sqrt{\frac{2}{\mathcal{A}}} \cos \left(G_{y} y / 2\right) c_{z 2} \mathrm{e}^{q_{2} z} \cos \left(G_{z} z / 2+\delta_{2}\right) \quad z<0$

where $\mathcal{A}$ is the surface area of the system and $c_{z i}$ is the normalization constant in the $z$ direction. The relation between the energies, $q_{j}$ and $\delta_{j}$, will be determined by the secular equation

$$
\left|\begin{array}{cc}
{\left[\left(G_{z}+2 \mathrm{i} q_{j}\right)^{2}+4 G_{y}^{2}\right] / 8 m^{*}-E_{j}} & V \\
V & {\left[\left(G_{z}-2 \mathrm{i} q_{j}\right)^{2}+4 G_{y}^{2}\right] / 8 m^{*}-E_{j}}
\end{array}\right|=0
$$

where $V$ is the lattice potential and $m^{*}$ is the effective mass. These were given in reference [16]. Following Smith [10], the wave functions outside of the crystal are solved for numerically using the JJJ potential [17] in which the effects of both crystal structure and image potential are included. We require the wave functions inside and outside to matchi.e., wave functions and their derivatives should be continuous-and thus determine the energies. We adjusted the potential parameter $z_{0}$ to make the resulting energies fit with experimental data. The result and also the parameters are listed in table 1. One might notice that the $z_{0}$ are different for even and odd states. This is not necessarily a deficiency of the 
two-band model. The even wave functions have most amplitude concentrated on atomic sites while the odd ones concentrate in interstitial space. This could result in different effective potentials outside.

Similarly we can calculate the Green's function with the two-band model. This method was evolved from Mahan's theory of photoemission [9] where plane waves were used. Now the energy gap has to be taken into account. Since we have essentially a one-dimensional potential, we can reduce the Green's function from a three-dimensional one to a onedimensional one. The two-band model gives a trigonometric form to the wave function in the direction parallel to the surface, as shown in equations (20) and (21). The fact that only the vertical transition is important further simplifies our task. So we have

$G\left(\boldsymbol{r}, \boldsymbol{r}^{\prime} ; E\right)=\sqrt{\frac{2}{\mathcal{A}}} \sin \left(G_{y} y / 2\right) g_{s}\left(z, z^{\prime} ; E\right)+\sqrt{2} \cos \left(G_{y} / 2 y\right) g_{c}\left(z, z^{\prime} ; E\right)$

where $g_{s(c)}\left(z, z^{\prime} ; E\right)$ is the one-dimensional Green's function. It has the form

$g_{s(c)}\left(z, z^{\prime} ; E\right)=\left[\theta\left(z-z^{\prime}\right) \Psi_{+}(z ; E) \Psi_{-}\left(z^{\prime} ; E\right)+\theta\left(z^{\prime}-z\right) \Psi_{-}(z ; E) \Psi_{+}\left(z^{\prime} ; E\right)\right] / W(E)$

where $W(E)$ is the Wronskian and $\Psi_{+(-)}(z ; E)$ is the solution of the one-dimensional Schrödinger equation satisfying the boundary condition for $z \rightarrow+\infty(-\infty)$. In the twoband model (the upper sign is for $g_{c}\left(z, z^{\prime} ; E\right)$ and the lower sign for $g_{s}\left(z, z^{\prime} ; E\right)$ )

$$
\Psi_{-}(z ; E)=\mathrm{e}^{-\mathrm{i} k z} \pm c_{1} \mathrm{e}^{\mathrm{i}\left(G_{z}-k\right) z} \quad z<0
$$

where

$$
k=p+\mathrm{i} q
$$

and the relation between $c_{1}$ and $E$ can be obtained by solving the secular equation

$$
\left|\begin{array}{cc}
\left(4 k^{2}+G_{y}^{2}\right) / 8 m^{*}-E & V \\
V & {\left[4\left(G_{z}-k\right)^{2}+G_{y}^{2}\right] / 8 m^{*}-E}
\end{array}\right|=0 .
$$

For $z>0, \Psi_{-}(z ; E)$ is solved for numerically using the JJJ potential. However, at $z=0$, the wave function outside should match with that in equation $(22)$. As for $\Psi_{+}(z ; E)$, it is also solved for numerically outside with the requirement that it vanishes as $z \rightarrow \infty$. Inside the crystal (similarly, the upper sign is for $g_{c}\left(z, z^{\prime} ; E\right)$ and the lower sign for $g_{s}\left(z, z^{\prime} ; E\right)$ )

$$
\Psi_{+}(z ; E)=\mathrm{e}^{\mathrm{i} k z} \pm c_{2} \mathrm{e}^{\mathrm{i}\left(k-G_{z}\right) z}+\beta \Psi_{-}(z ; E) \quad z<0
$$

where $k$ and $c_{2}$ are related to $E$ by equation (24). Coefficient $\beta$ enables $\Psi_{+}(z ; E)$ inside to match with that outside.

We would consider the second-harmonic generation resonant with $\psi_{1}(r)$ and $\psi_{2}(r)$, i.e., $\hbar \omega \simeq E_{2}-E_{1}$. Only the resonance terms were taken into account. Going back to the last section, we found that

$$
J_{1}=J_{2}=0
$$

in the case of vertical transition and resonance. This is because in equations (12) and (13), the intermediate state will be $\psi_{2}(\boldsymbol{r})$, which has parity different from that of $\psi_{1}(\boldsymbol{r})$. For the second-harmonic generation, $J_{1}$ and $J_{2}$ should be constant on the atomic scale because we are working in the long-wavelength region. The product of $\psi_{1}(\boldsymbol{r})$ and $\psi_{2}(\boldsymbol{r})$ which appears 
in $J_{1}$ and $J_{2}$ has a wavelength of a lattice constant and thus cannot produce the second harmonics that we want. Substituting equations (17), (20), and (21) into (16), we get

$$
\begin{aligned}
J_{3, y}(\boldsymbol{r}, t)= & \frac{\mathrm{i} e^{3} G_{y}^{2} A_{0, y} A_{0, z} D}{4 \mathcal{A} m^{3} c^{2}} \mathrm{e}^{-2 \mathrm{i} \omega t} \int \mathrm{d} z^{\prime} \int \mathrm{d} z^{\prime \prime}\left[Z_{1}(z) g_{c}\left(z, z^{\prime} ; 2 \hbar \omega+E_{1}+\mathrm{i} \eta\right)\right. \\
& \times \frac{\mathrm{d}}{\mathrm{d} z^{\prime}} g_{c}\left(z^{\prime}, z^{\prime \prime} ; \hbar \omega+E_{1}+\mathrm{i} \eta\right) Z_{1}\left(z^{\prime \prime}\right) \\
& \left.+Z_{1}\left(z^{\prime}\right) \frac{\mathrm{d}}{\mathrm{d} z^{\prime}} g_{s}\left(z^{\prime}, z ;-\hbar \omega+E_{1}-\mathrm{i} \eta\right) g_{c}\left(z, z^{\prime \prime} ; \hbar \omega+E_{1}+\mathrm{i} \eta\right) Z_{1}\left(z^{\prime \prime}\right)\right]
\end{aligned}
$$

and

$$
\begin{aligned}
J_{3, z}(\boldsymbol{r}, t)=\frac{\mathrm{i} e^{3} G_{y}^{2} A_{0, y}^{2} D}{4 \mathcal{A} m^{3} c^{2}} \mathrm{e}^{-2 \mathrm{i} \omega t} \int \mathrm{d} z^{\prime} \int \mathrm{d} z^{\prime \prime}\left[Z_{1}(z)\right. \\
\quad \times \frac{\mathrm{d}}{\mathrm{d} z} g_{s}\left(z, z^{\prime} ; 2 \hbar \omega+E_{1}+\mathrm{i} \eta\right) g_{c}\left(z^{\prime}, z^{\prime \prime} ; \hbar \omega+E_{1}+\mathrm{i} \eta\right) Z_{1}\left(z^{\prime \prime}\right) \\
\left.\quad+Z_{1}\left(z^{\prime}\right) g_{c}\left(z^{\prime}, z ;-\hbar \omega+E_{1}-\mathrm{i} \eta\right) \frac{\mathrm{d}}{\mathrm{d} z} g_{c}\left(z, z^{\prime \prime} ; \hbar \omega+E_{1}+\mathrm{i} \eta\right) Z_{1}(z)\right]
\end{aligned}
$$

where

$$
D=\frac{m}{\pi \hbar^{2}} \times 7.35 \times 10^{-3} \mathrm{Ryd} .
$$

In fact $D$ is the product of the surface density of states $m / \pi \hbar^{2}$ and the energy (in atomic units) by which the lower surface state is below the Fermi level. At this point it is appropriate to discuss the effect of the effective mass. It is not important because each Green's function provides one factor of the effective mass. Together with $D$, they completely cancelled the effective mass to the third power in the denominator of the current density. Now the current density can readily be evaluated after evaluating $\Psi_{ \pm}(\boldsymbol{r})$.

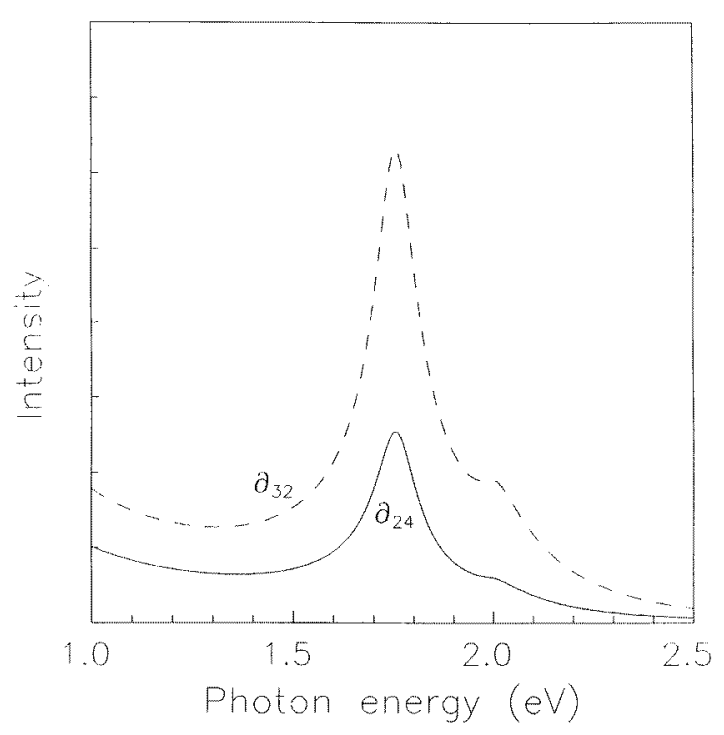

Figure 1. $\partial_{24}$ (the solid line) and $\partial_{32}$ (the dashed line) as functions of the fundamental frequency. 


\section{Reflectance}

What is usually measured is the reflection intensity of the second-harmonic generation. In principle, this can be done with Maxwell equations. To simplify the matter, we assume that the nonlinear current and hence the polarization concentrates at the surface [11]:

$$
\boldsymbol{P}(\boldsymbol{r}, t)=\boldsymbol{I} \boldsymbol{I} \delta(z) \mathrm{e}^{2 \mathrm{i}(\boldsymbol{u} \cdot \boldsymbol{r}-\omega t)}
$$

where

$$
\begin{aligned}
& I I_{x}=2 \partial_{15} E_{i n, x} E_{i n, z} \\
& I I_{y}=2 \partial_{24} E_{i n, y} E_{i n, z}
\end{aligned}
$$

and

$$
I I_{z}=\partial_{31} E_{i n, x}^{2}+\partial_{32} E_{y}^{2}+\partial_{33} E_{i n, z}^{2}
$$

with

$$
E_{\text {in }}=\mathrm{i} \omega A_{0} / c
$$

for a fcc (110) crystal surface. In view of the relation $\boldsymbol{J}=\dot{\boldsymbol{P}}$, equations (26)-(28), we found

$$
\begin{aligned}
& \partial_{15}=\partial_{31}=\partial_{33}=0 \\
& \begin{aligned}
& \partial_{24}=\frac{e^{3} G_{y}^{2} D}{16 m^{3} \omega^{3}} \int \mathrm{d} z \int \mathrm{d} z^{\prime} \int \mathrm{d} z^{\prime \prime}\left[Z_{1}(z) g_{c}\left(z, z^{\prime} ; 2 \hbar \omega+E_{1}+\mathrm{i} \eta\right)\right. \\
& \quad \times \frac{\mathrm{d}}{\mathrm{d} z^{\prime}} g_{c}\left(z^{\prime}, z^{\prime \prime} ; \hbar \omega+E_{1}+\mathrm{i} \eta\right) Z_{1}\left(z^{\prime \prime}\right) \\
&+\left.Z_{1}\left(z^{\prime}\right) \frac{\mathrm{d}}{\mathrm{d} z^{\prime}} g_{s}\left(z^{\prime}, z ;-\hbar \omega+E_{1}-\mathrm{i} \eta\right) g_{c}\left(z, z^{\prime \prime} ; \hbar \omega+E_{1}+\mathrm{i} \eta\right) Z_{1}\left(z^{\prime \prime}\right)\right]
\end{aligned}
\end{aligned}
$$

and

$$
\begin{aligned}
\partial_{32}=\frac{e^{3} G_{y}^{2} D}{8 m^{3} \omega^{3}} & \int \mathrm{d} z \int \mathrm{d} z^{\prime} \int \mathrm{d} z^{\prime \prime}\left[Z_{1}(z)\right. \\
& \times \frac{\mathrm{d}}{\mathrm{d} z} g_{s}\left(z, z^{\prime} ; 2 \hbar \omega+E_{1}+\mathrm{i} \eta\right) g_{c}\left(z^{\prime}, z^{\prime \prime} ; \hbar \omega+E_{1}+\mathrm{i} \eta\right) Z_{1}\left(z^{\prime \prime}\right) \\
& \left.+Z_{1}\left(z^{\prime}\right) g_{c}\left(z^{\prime}, z ;-\hbar \omega+E_{1}-\mathrm{i} \eta\right) \frac{\mathrm{d}}{\mathrm{d} z} g_{c}\left(z, z^{\prime \prime} ; \hbar \omega+E_{1}+\mathrm{i} \eta\right) Z_{1}(z)\right] .
\end{aligned}
$$

By taking $\eta$ to be $0.1 \mathrm{eV}$ which seems to be close to the experimental resolution, we calculated $\partial_{24}$ and $\partial_{32}$. These are plotted in figure 1 as functions of $\omega$. The peak at $1.75 \mathrm{eV}$ is the resonance of the surface-state-surface-state transition while the structure at $2 \mathrm{eV}$ indicates the onset of the upper band edge. Notice that $\partial_{32}$ is greater than $\partial_{24}$. The reason for this is that the upper-band wave function has a $z$-dependence very different from that of the lower-band wave function. The lower surface state and its wave function are quite close to the lower band ( $q_{1}$ in equation (17) is very small). In $J_{y}$ and hence $\partial_{24}, \psi_{1}$ coupled with the upper band through $g_{c}$. Therefore, the matrix is small in magnitude. In $J_{z}$ and $\partial_{32}$ there is a $z$-derivative operator in between. This results in a larger $J_{z}$.

It is worth comparing the results with those of the jellium model. According to Rudnick and Stern [18]

$$
P_{y(x)}=\frac{\mathrm{i} b e^{3} n}{m^{2} \omega^{4}} E_{i n, y(x)} E_{i n, z}
$$


and

$$
P_{z}=\frac{\mathrm{i} a e^{3} n}{2 m^{2} \omega^{4}} E_{\mathrm{i} n, z}^{2}
$$

where $n$ is the electron density and $a$ and $b$ are parameters (or slowly varying functions of frequency). We immediately see that $\partial_{24}$ and $\partial_{32}$ have different frequency dependences from those in the jellium model. This is because those from reference [18] were derived in the high-frequency limit. In that case, $\boldsymbol{J}_{3}(\boldsymbol{r}, t)$ is negligible compared to $\boldsymbol{J}_{1}(\boldsymbol{r}, t)$ and $\boldsymbol{J}_{2}(\boldsymbol{r}, t)$ and equations (38) and (39) can be derived from equations (12) and (13). On the other hand, $\partial_{24}$ and $\partial_{32}$ come from $\boldsymbol{J}_{3}(\boldsymbol{r}, t)$. Nevertheless, it is important to see how the secondharmonic generation from resonance is compared with that from jellium in magnitude - that is to say, if it is detectable in experiments. From our calculation we found that

$$
\frac{m^{2} \omega^{4}}{e^{3} n}\left|\partial_{24}\right|:|b| \simeq 10:|b|
$$

at the peak position. Since $|b|$ is of the order of unity, the resonance signal is clearly visible.

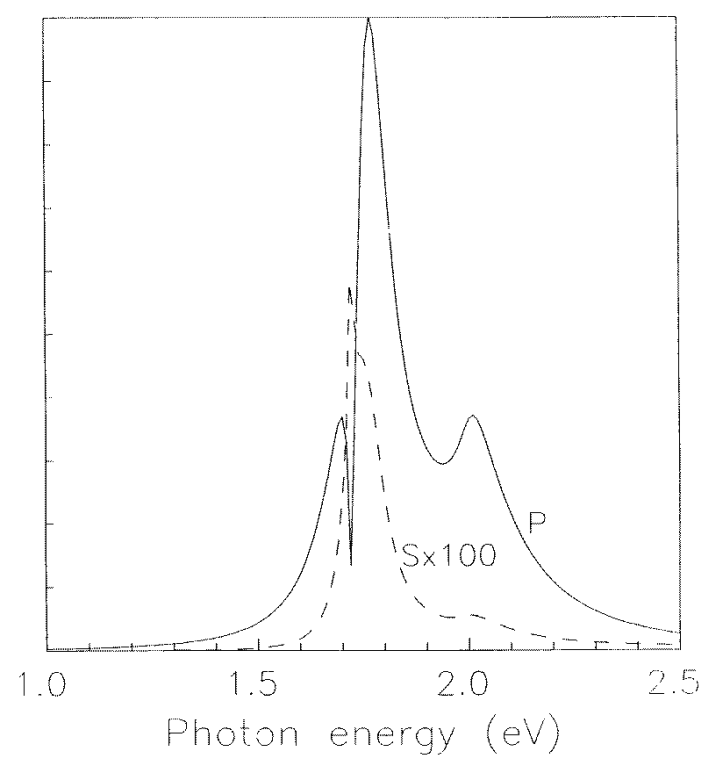

Figure 2. The reflection intensity of the second harmonic induced by p-polarized incident light. The intensity of the p-polarized second harmonics is shown by the solid line and that of the s-polarized light multiplied by 100 is shown as the dashed line.

Using the boundary conditions of electric and magnetic fields accounting for surface current and polarization, or following Sipe et al [11], for s-polarized incident light, there is no reflected s-polarized second-harmonic light. For the reflected p-polarized secondharmonic light

$$
|\boldsymbol{E}|=\frac{8 \pi \mathrm{i} \epsilon(2 \omega) \kappa_{\|} \omega \partial_{32}}{c\left[\kappa_{0} \epsilon(2 \omega)+\kappa_{2}\right]} E_{i n}^{2} \sin ^{2} \phi
$$

where $\phi$ is the angle between the incident plane and the $y$-direction. For p-polarized incident light, the reflected s-polarized light has

$$
|\boldsymbol{E}|=\frac{-16 \pi \mathrm{i} \kappa_{||} \kappa_{1} \partial_{24}}{\epsilon(\omega)\left(\kappa_{0}+\kappa_{2}\right)} E_{i n}^{2} \sin \phi \cos \phi
$$




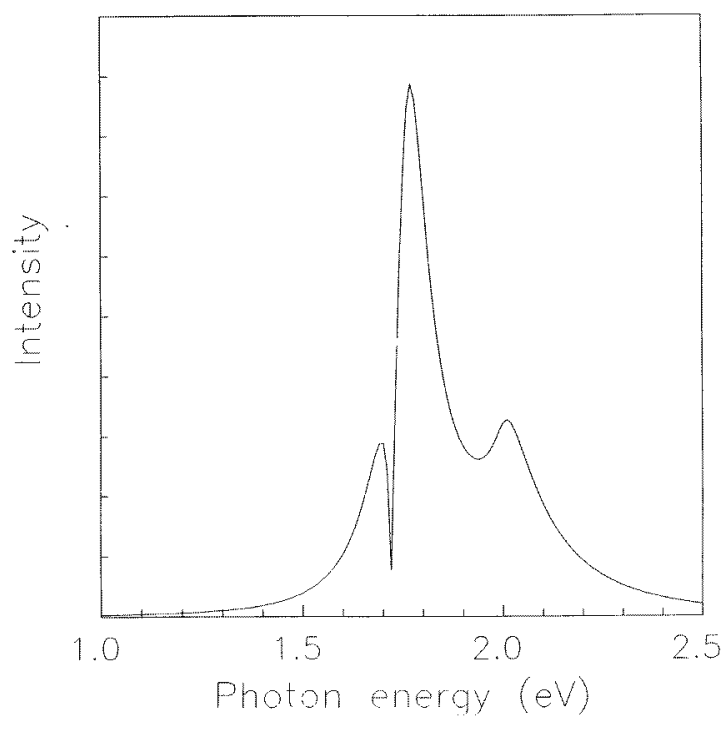

Figure 3. The reflection intensity of the ppolarized second harmonics induced by $\mathrm{s}$ polarized incident light.

and the p-polarized light has

$$
|\boldsymbol{E}|=\frac{8 \pi \mathrm{i} c \kappa_{||}\left[\kappa_{1}^{2} \epsilon(2 \omega) \partial_{32}-2 \kappa_{2} \kappa_{1} \partial_{24}\right]}{\omega \epsilon(\omega)\left[\kappa_{0} \epsilon(2 \omega)+\kappa_{2}\right]} E_{i n}^{2} \cos ^{2} \phi .
$$

Here, we defined

$$
\begin{aligned}
\kappa_{\|} & =\frac{\omega}{c} \sin \theta \\
\kappa_{0} & =\sqrt{\omega^{2} / c^{2}-\kappa_{\|}^{2}} \\
\kappa_{1} & =\sqrt{\omega^{2} \epsilon(\omega) / c^{2}-\kappa_{\|}^{2}} \\
\kappa_{2} & =\sqrt{\omega^{2} \epsilon(2 \omega) / c^{2}-\kappa_{\|}^{2}}
\end{aligned}
$$

where $\theta$ is the angle of incidence and $\epsilon(\omega)$ is the dielectric function. To calculate the reflection intensity, we need to know the dielectric function. We fitted the experimental data of Johnson and Christy [19] with a two-parameter function. The dielectric function consists of a Drude part and an interband transition part. The imaginary part of the latter is of the form [20] $\xi \theta\left(\omega-e_{d}\right) \sqrt{\omega-e_{d}} / \omega^{2}$. The real part was found from the Kramers-Kronig relation. Thus

$$
\epsilon(\omega)=1-\frac{\omega_{p}^{2}}{\omega(\omega+\mathrm{i} / \tau)}-\frac{\xi\left(2 \sqrt{e_{d}}-\sqrt{e_{d}+\omega}-\sqrt{e_{d}-\omega}\right)}{\omega^{2}} .
$$

For $\tau$ and the optical effective mass, we used the values of reference [17] where $m^{*}$ is equal to the free-electron mass and $1 / \tau=31 \times 10^{15} \mathrm{~s}^{-1}$. The two parameters are $\xi=18 \mathrm{eV}^{-3 / 2}$ and $e_{d}=3.45 \mathrm{eV}$. This simple form gives a fairly good fit and reproduces all of the important features. With this we calculated the reflection intensity of the second harmonics. In figure 2, the intensity of p-polarized light and s-polarized light times 100 induced by p-polarized incident light are shown as solid and dashed curves respectively. In figure 3, the intensity of p-polarized light induced by s-polarized incident light is plotted. The unit of intensity is arbitrary but it is the same in both figures. We can see that the intensity of 
p-polarized light is higher than that of s-polarized light. This is mainly because $\partial_{32}$ is larger than $\partial_{24}$ in magnitude. The fact that $\epsilon(\omega)$ appears in the denominator of equation (42) also plays a role. The main peaks at $1.75 \mathrm{eV}$ in both figures come from the resonance between two surface states. The structures at $2 \mathrm{eV}$ have the same origin as that in figure 1 . The dips at $1.7 \mathrm{eV}$ show the dielectric effect. It comes from $\epsilon(2 \omega)$ in the numerators of equations (41) and (43). At $2 \omega=e_{d}$, it approaches zero. But experimentally it might be difficult to detect because the dip is very narrow.

The experiment of Urbach et al [2] provides a direct comparison. They measured the second-harmonic intensity in the cases of the incident plane parallel and normal to the $y$ direction. They found no s-polarized second-harmonic light. This is expected because $\phi$ in equation (42) is either 0 or $\pi / 2$. For p-polarized second-harmonic light, there is the main peak at $1.75 \mathrm{eV}$ and some structure at around $2 \mathrm{eV}$. Both were predicted by our theory. Our results do not have a peak at $2 \mathrm{eV}$ as shown in their figure 1(a) with squares because we did not take into account the surface-state-bulk-band transition. The Green's functions were calculated in the presence of the JJJ surface potential. The relative intensity needs more study. In reference [2] the ratio of the second-harmonic signals produced by p-polarized incident light to those produced by the s-polarized light is about 6 while in our results it is about 1 . We agree that most of the contribution comes from $\partial_{32}$, but we attribute the discrepancy in ratio to the enhancement of the electric field normal to the surface of the fundamental wave by conduction electrons. For the same incident light intensity the p-polarized fundamental wave inside the crystal has a much stronger electric field [5-8] than that of the s-polarized wave. Our theory calculated the second-harmonic current based on the electric field inside the crystal. To convert it to the electric field of the incident light, $\partial_{24}$ should be multiplied by an enhancement factor. The calculation of this factor is beyond the scope of the present work.

\section{Conclusion}

We have developed a simple and intuitive formalism for analysing the second-harmonic generation induced by surface states. The energy gap and all of the intermediate states are taken into account properly. It is applied to the silver (110) surface. The results fit the experiments reasonably well. This method can easily be generalized to incorporated more bands. It also provides a basis on which to consider many-body effects such as screening and surface plasmons. We have calculated $\partial_{32}$ and $\partial_{24}$. Both are are greater in magnitude by at least a factor of 10 than the output from jellium at resonance. Hence, the signal is clearly observable. Comparing them with each other, $\partial_{32}$ is at least four times greater than $\partial_{24}$. The ratio can be as large as 10 at resonance. Experimentally one can identify the source of the second-harmonic generation (from either $\partial_{32}$ or $\partial_{24}$ ) because $\partial_{32}$ is coupled to $E_{y}^{2}$ and $\partial_{24}$ is coupled to $E_{y} E_{z}$. However, $E_{z}$ is enhanced inside the metal. Though we did not calculate the enhancement factor, we can estimate it from experimental data. For the second-harmonic generation near resonance, the dielectric effect is dominated by the system response, at least for experiments. In figures 2 and 3, the main features come from the response. The dips given by the dielectric function were not detected by experiments.

\section{Acknowledgment}

This work was supported in part by the NSC of Taiwan, Republic of China, under the contract number NSC83-0208-M-002-050. 


\section{References}

[1] Shen Y R 1984 The Principles of Nonlinear Optics (New York: Wiley)

[2] Urbach L E, Percival K L, Hicks J M, Plummer E W and Dai H-L 1992 Phys. Rev. B 453769

[3] Li C M, Urbach L E and Dai H-L 1994 Phys. Rev. B 492104

[4] Lüpke G, Bottomley D J and van Driel H M 1994 Phys. Rev. B 4917303

[5] Schaich W L and Liebsch A 1988 Phys. Rev. B 376187

[6] Weber M and Liebsch A 1987 Phys. Rev. B 357411

[7] Liebsch A 1988 Phys. Rev. Lett. 611233

[8] Liebsch A and Schaich W L 1989 Phys. Rev. B 405401

[9] Mahan G D 1970 Phys. Rev. B 24334

[10] Smith N V 1985 Phys. Rev. B 323549

[11] Sipe J E, Moss D J and van Driel H M 1987 Phys. Rev. B 351129

[12] Bower J R 1976 Phys. Rev. B 142427

[13] Hu C D 1989 Phys. Rev. B 407520

[14] Hu C D 1993 Phys. Rev. B 477525

[15] See, for example, Chen C T and Smith N V 1987 Phys. Rev. B 355407

Kevan S D 1986 Phys. Rev. B 346713

Kevan S D, Stoffel N G and Smith N V 1985 Phys. Rev. B 313348

[16] Smith N V, Chen C T and Weinert M 1989 Phys. Rev. B 407565

[17] Jones R O, Jennings P J and Jepsen O J 1984 Phys. Rev. B 296474

[18] Rudnick J and Stern E A 1971 Phys. Rev. B 44274

[19] Johnson P B and Christy R W 1972 Phys. Rev. B 64370

[20] Harbeke G 1972 Optical Properties of Solids ed F Abelès (Amsterdam: North-Holland) ch 2 Abelès F 1972 Optical Properties of Solids ed F Abelès (Amsterdam: North-Holland) ch 3 